\title{
Estimation of losses per unit length in a rectangular waveguide with rough screening surfaces based on the concept of partial waves
}

\author{
Vladimir Biryukov ${ }^{1,}$, Vladimir Grachev ${ }^{1}$, Ekaterina Karakozova $^{1}$, Sergey Lobin ${ }^{2}$, and \\ Vladimir Shcherbakov \\ ${ }^{1}$ Nizhny Novgorod State Technical University n.a. R.E. Alekseev, Minin St., 24, Nizhny Novgorod, \\ 603950, Russia \\ ${ }^{2}$ Branch of Federal State Unitary Enterprise "Russian Federal Nuclear Center-All-Russian Research \\ Institute of Experimental Physics" "Research Institute of Measuring Systems n. a. Yu.Ye. Sedakov", \\ Tropinin St., 47, Nizhny Novgorod, 603137, Russia
}

\begin{abstract}
A method for calculating the rough surface reflection coefficient of an electromagnetic wave is proposed. It is shown that the screening surface roughness in waveguides is equivalent to a decrease in conductivity of these surfaces in comparison with values belonging to Schukin-Leontovich boundary conditions for completely smooth surfaces. Examples of calculating the attenuation coefficient in the rectangular waveguide with rough screening surfaces in a terahertz frequency range are presented. The influence of the size and shape of the rough surface profile irregularities on the waveguide attenuation is studied.
\end{abstract}

\section{Introduction}

The screening surface imperfection is the main factor determining the losses per unit length of the guide electrodynamic structure at high frequencies. This imperfection appeared as finite conductivity in the material and screening surface roughness, determined by the quality of its processing. For this reason, both theoretical [1-4] and experimental [5-6] studies have recently been devoted to investigating the influence of surface roughness on the characteristics of guiding electrodynamic structures.

The surface roughness can be neglected if its size is much smaller than the penetration depth of the electromagnetic field into the screen walls. This condition is usually satisfied at frequencies below and on the order of hundreds of megahertz. However, as the frequency increases, requirements for the quality of screening surface processing are increased and become comparable with technological capabilities. In this regard, in the terahertz frequency range the screening surface roughness becomes the main factor determining the losses per unit length in the guide structure.

In this paper, the influence of the profile height and shape of the screening surface roughness on the main wave attenuation per unit length in the rectangular waveguide is studied. A rough surface is considered, i.e. it is assumed that the mirror component of the reflected wave is much larger than the diffusion wave.

*Corresponding author: birukovvv@mail.ru 


\section{Method for estimating the losses per unit length in the waveguide}

We consider the case when roughness size is comparable with skin depth, but much less than the wavelength propagating in the waveguide.

In this paper, an approach based on the concept of Brillouin partial waves is proposed. Basically, it allows for taking into account the random surface roughness of the free form. Since the field of any wave in the waveguide is the result of a superposition of plane waves repeatedly reflected from the screening surface, its attenuation is determined by the magnitude of the reflection coefficient and the number of reflections. If the conducting surface is completely smooth, the module of the reflection coefficient is equal to one, and the partial-wave interference occurs in phase. As a result, a natural wave propagates in the waveguide without attenuation.

Wave attenuation appears either when the module of the reflection coefficient decreases, when during partial-wave interference phase relationships are changed, or if both of these factors are present. The first case corresponds to an imperfectly conducting screening surface and has been well studied. The second case that occurs in a waveguide with smooth walls corresponds to reactively incident waves.

Partial scattering occurs in a waveguide with rough walls during the reflection of partial waves, as a result, phase relations are changed according to the secondary wave interference. Part of the secondary waves don't participate in the waveguide's natural wave formation, thereby taking away part of the energy from it. As a result, propagating natural waves becomes incident even in the case of perfectly conductive screening surfaces.

The problem of the electromagnetic wave scattering on statistically rough surfaces is studied in radiolocation, fluoroscopy, and optics. To apply the obtained results to the estimation the natural wave attenuation of the guiding electrodynamic structures, it is proposed to find the parameters of an equivalent smooth surface having the same reflection coefficient as a real rough surface. Thus, wave attenuation caused by surface roughness can be found, for example, by correcting the conductivity of the screen material.

In this case, the attenuation $\alpha$ in the waveguide depends on the reflection coefficient $\Gamma$ of the electromagnetic wave from the waveguide wall and the number of reflections:

$\alpha=20 \log |\Gamma|^{n}$, where $n$ - number of reflections. The number of reflections of the main wave in a rectangular waveguide $l$ can be defined using the expression:

$$
n=\frac{l}{a \sqrt{\left(\frac{2 a f}{c}\right)^{2}-1}},
$$

where $a$ - the size of the waveguide wide wall.

Expressions for reflection coefficients of mutually perpendicular components of the electric field intensity ( $\Gamma_{||}$for lying in the plane of incidence and $\Gamma_{\perp}$ for the components perpendicular to this plane) can be obtained using well-known Fresnel formulas.

In view of the above, the expressions for the attenuation coefficients of the electromagnetic field components in a rectangular waveguide can be represented as:

$$
\alpha_{||}=\frac{20 \pi l}{a^{2} \sqrt{\left(\frac{\omega}{c}\right)^{2}-\left(\frac{\pi}{a}\right)^{2}}} \log \Gamma_{||},
$$




$$
\alpha_{\perp}=\frac{20 \pi l}{a^{2} \sqrt{\left(\frac{\omega}{c}\right)^{2}-\left(\frac{\pi}{a}\right)^{2}}} \log \Gamma_{\perp} .
$$

In the case of the smooth screening surface, the obtained frequency dependence of the attenuation coefficient both qualitatively and quantitatively is the same as standard.

But in addition to the losses related to the final conductivity of the waveguide walls, losses related to the quality of screening surface processing can also have a significant influence, especially when the signal frequency under consideration is increased. In this paper, we propose using an approach based on the concept of partial Brillouin waves in order to take into account the surface roughness of the free form, including a random one. In this case, the problem of finding the natural wave attenuation in the guiding electrodynamic structures with rough screening surfaces is reduced to the case of structures with completely smooth surfaces. The equivalence of the real rough surface and smooth surface is achieved by correcting the conductivity of the latter material, ensuring the equality of the reflection coefficients of the electromagnetic wave from the surfaces under consideration.

\section{Rough conductive surface reflection coefficient}

The profile shape of the rough surface depends on the processing method and tool used. This profile shape is random in nature and is described by statistical surface characteristics.

The reflection of a plane electromagnetic wave from a statistically uneven conductive surface is considered. The equation of a stationary surface can be specified by a random function $x=\xi(y, z)$ two variables where the axis $« z$ » coincides with the axis of the waveguide. This function is assumed to be single-valued and sufficiently smooth (a sufficient number of times differentiable with both arguments). The field reflected from such a surface is random. Not the field itself, but the random surface ensemble average reflection coefficient is important.

Strictly speaking, any random variable is statistically completely described by an ndimensional probability density for $n \rightarrow \infty$. However, in our case, the one-dimensional probability density $w_{1}(x, y, z)$ is proposed. It can show that the surface height $\xi$ at the point with coordinates $y, z$ lies within $x<\xi(y, z)<x+d x$. One-dimensional probability density allows finding the average value of a random variable and the standard deviation of a random variable or its function from the average value.

In the case of a spatially homogeneous random variable, the one-dimensional probability density is the same almost everywhere

$$
w_{1}(x, y, z)=w_{1}(x)=w_{1}(\xi) .
$$

The average surface height the above the plane $x=0$ is defined by the expression

$$
\bar{\xi}=\int_{-\infty}^{\infty} \xi w_{1}(\xi) d \xi
$$

The surface height scatter relative to the average value is described by the standard deviation $\delta$ (the line above indicates static averaging)

$$
\delta^{2}=\overline{(\xi-\bar{\xi})^{2}}=\int_{-\infty}^{\infty}(\xi-\bar{\xi})^{2} w_{1}(\xi) d \xi
$$


These characteristics describe random variables that follow Gaussian law. If surface roughness is caused by a large number of independent factors, then, based on the central limit theorem of probability law, such a surface is described by Gaussian law.

$$
w_{1}(\xi)=\frac{1}{\delta \sqrt{2 \pi}} \exp \left(-\frac{(\xi-\bar{\xi})^{2}}{2 \delta^{2}}\right) .
$$

Since a reflected field is created due to the interference of an infinitely large number of waves reflected from elements in the random surface, by virtue of the central limit theorem, it also follows the Gaussian law.

In a first approximation, the difference between the reflection of a plane electromagnetic wave from the rough and smooth surfaces is that in the case of a smooth surface the phases of the secondary waves are known and determined by the phase of the incident wave. With the static rough surface the phases of the secondary waves differ from the values determined by the incident wave by random values (Fig. 1)

$$
\Delta \varphi(\xi)=k_{0} \cdot 2 \xi \cdot \cos \theta=\frac{2 \pi}{\lambda_{0}} 2 \xi \cdot \cos \theta,
$$

where $\theta$ - angle of incidence.

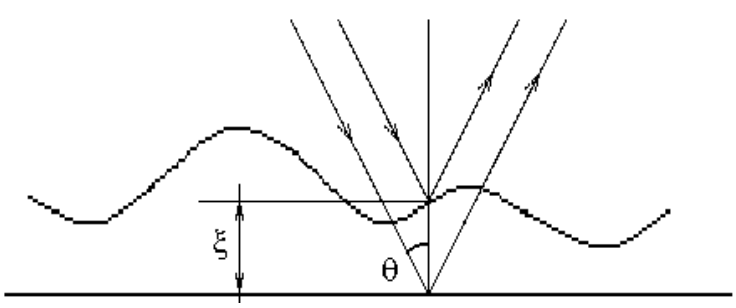

Fig. 1. The influence of the random rough surface height on the phase of the reflected wave.

In this case, the random complex amplitude of the electric field intensity of the reflected wave can be represented as

$$
E_{\text {отр }}=E_{\text {пад }} \Gamma(\theta) \exp \left(i k_{0} 2 \xi \cos \theta\right)
$$

where $\Gamma(\theta)$ - smooth surface reflection coefficient.

Its average value is determined by the expression

$$
\bar{E}_{\text {отр }}=E_{\text {пад }} \Gamma(\theta) \int_{-\infty}^{\infty} \exp \left(i k_{0} 2 \xi \cos \theta\right) w(\xi) d \xi .
$$

Introducing probability density here, we obtain the average rough surface reflection coefficient

$$
\bar{\Gamma}(\theta)=\Gamma(\theta) \exp \left(-8 \pi^{2} \cos ^{2} \theta\left(\frac{\delta}{\lambda_{0}}\right)^{2}\right) .
$$

According to the last expression, the rough surface reflection coefficient depends on the roughness size relative to the wavelength. Figure 2 shows the dependence of the normalized 
reflection coefficient on the relative roughness size for two values of the angle of incidence. The reflection coefficient is normalized to the value of the smooth surface reflection coefficient.

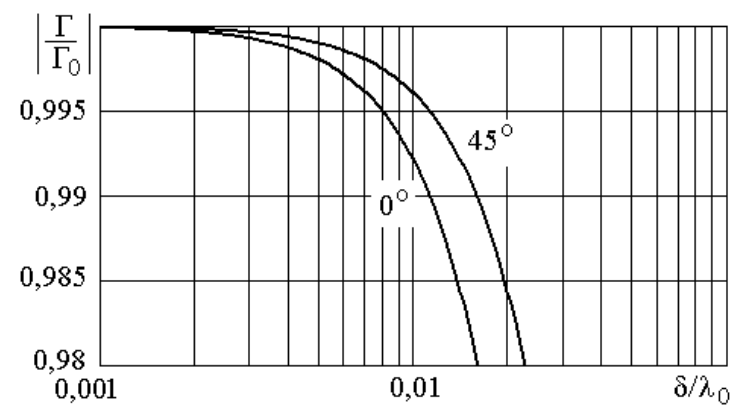

Fig. 2. Dependence of the normalized rough surface reflection coefficient on the roughness size related to the wavelength at the angle of incidence 0 and $45^{\circ}$.

At first glance, noticeable changes in the reflection coefficient begin with roughness sizes exceeding one hundredth of a wavelength. However, as the following graph shows (Fig. 3), the reflection coefficient decrease by thousandths is equivalent to a decrease in the conductivity of a smooth surface by almost an order of magnitude. In addition, it should be noted that the maximum values of the random variable are 5-6 times larger than the rootmean- square value with Gaussian distribution.

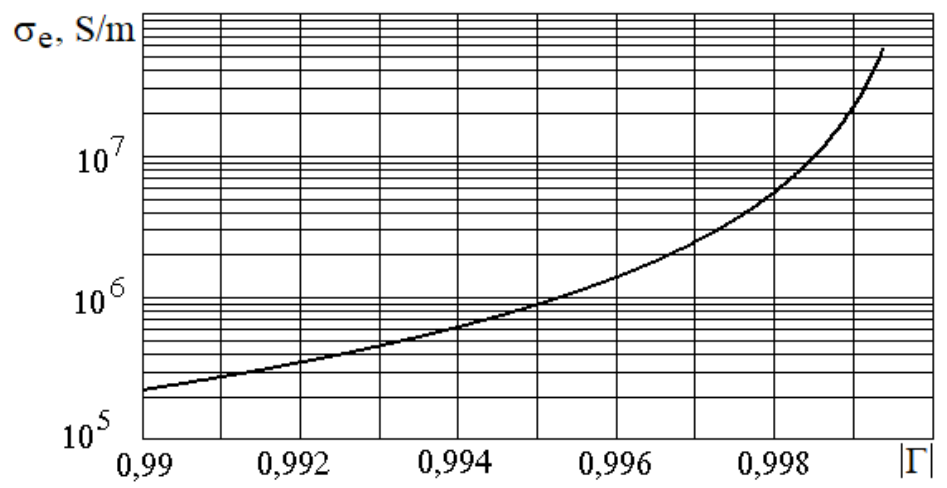

Fig. 3. Dependence of equivalent surface conductivity on the module of reflection coefficient.

Thus, the influence of roughness is significant when its size is thousandths of a wavelength.

\section{Estimating results for losses per unit length in a rectangular waveguide}

Figure 4 shows the dependences on the frequency loss of a rectangular waveguide with a standard cross section of $1.1 / 0.55 \mathrm{~mm}$ for smooth copper walls and with roughness of 3 , 10 , and $20 \mu \mathrm{m}$, accordingly, following the Gaussian law. According to the chart comparison, roughness of $3 \mu \mathrm{m}$ practically does not increase the losses per unit length in the waveguide. Roughness of 10 microns increases losses almost twice and roughness of 20 microns increases almost 4 times. Losses in the latter case are approximately $30 \mathrm{~dB} / \mathrm{m}$, while the roughness is 50 times smaller than the wavelength. 


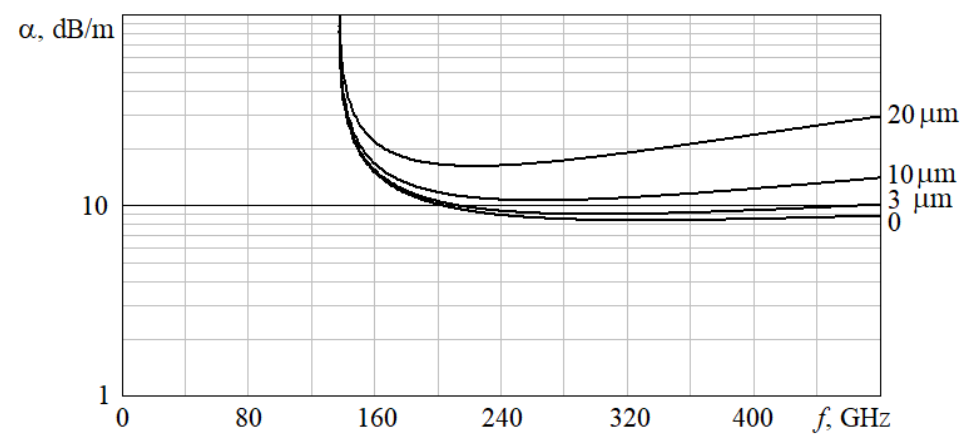

Fig. 4. Dependence on the frequency of the attenuation coefficient in the rectangular waveguide with the cross section of $1.1 / 0.55 \mathrm{~mm}$ for copper walls with different roughness size.

As a model of rough surface, we consider periodically repeating profile irregularities in various shapes. In the perpendicular direction, the profile is considered to be unchanged.

In this approximation, the surface profile is described by a function of one variable $\xi=\xi(x),-B<x<B$. The probability density in this case is represented as the relative interval of the profile height within $\xi, \xi+d \xi$.

Assuming formally $\xi(x)$ random function, we can use the well-known expression relating the probability density of a random function and its argument:

$$
\omega_{\xi}(\xi)=\omega_{x}\left(\xi^{-1}(\xi)\left|\frac{\partial \xi^{-1}}{\partial \xi}(\xi)\right| .\right.
$$

We find the dependencies for rectangular, piecewise linear and smooth profiles.

In the case of rectangular shape irregularities with a height $A$ and a transverse size $D$, the probability density has the form:

$$
\omega_{\xi}(\xi)=\frac{B-D}{B} \delta(\xi)+\frac{D}{B} \delta(\xi-A)
$$

where $\delta(\xi)$ - delta function or Dirac function.

Figure 5 shows the estimation results for the losses per unit length of a rectangular waveguide with a cross section of $1.1 \times 0.55 \mathrm{~mm}$ having a rectangular profile for different values of the profile height.

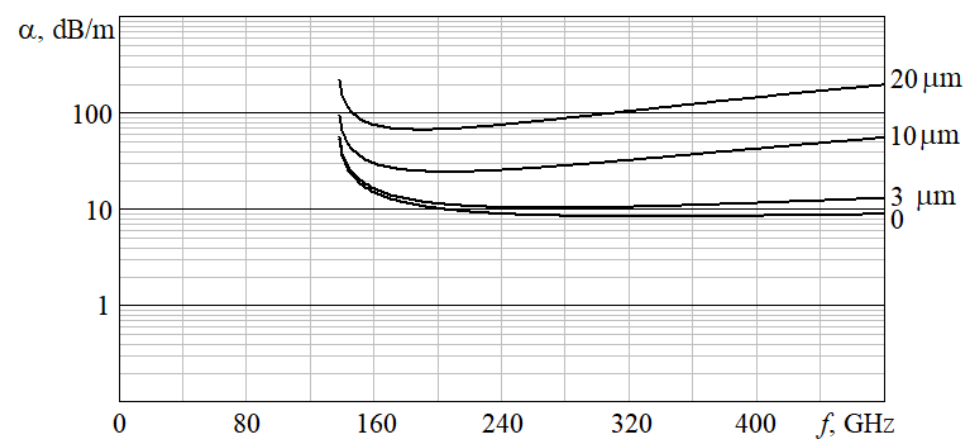

Fig. 5. Dependence on the frequency of losses per unit length in the rectangular waveguide with a cross section of $1.1 \times 0.55 \mathrm{~mm}$ having a rectangular profile for a relative profile bearing length of $50 \%$. 
The dependences above show that the presence of surface roughness can lead to an increase in the attenuation per unit length in the waveguide by almost an order of magnitude. The greatest attenuation is observed when the transverse size is half the period of irregularities.

With a piecewise linear profile shape, the expression for the probability density takes the form:

$$
\omega_{\xi}(\xi)=\frac{B-D}{D} \delta(\xi)+\frac{D}{A B}
$$

The estimation results for the losses per unit length in a rectangular waveguide with a cross section of $1.1 \times 0.55 \mathrm{~mm}$ for a piecewise linear profile with various profile heights are presented in Fig. 6.

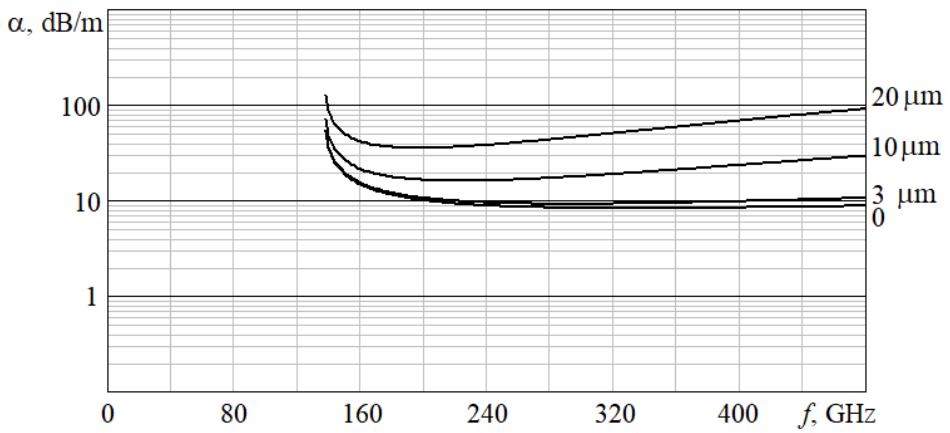

Fig.6. The dependence on the frequency of losses per unit length in a rectangular waveguide is $1.1 \times 0.55 \mathrm{~mm}$ with a linear profile for a relative profile bearing length of $50 \%$.

This comparison shows that at the same height of the irregularities the increase in attenuation in the case of a rectangular profile is greater than in the case of a linear one.

To describe a smooth profile the following function can be used $A\left|\cos ^{m}(x)\right|$, where the parameter $m$ can vary, allowing you to simulate a different relative profile bearing length. The profile view for various values of the parameter $m$ is shown in Fig. 7.

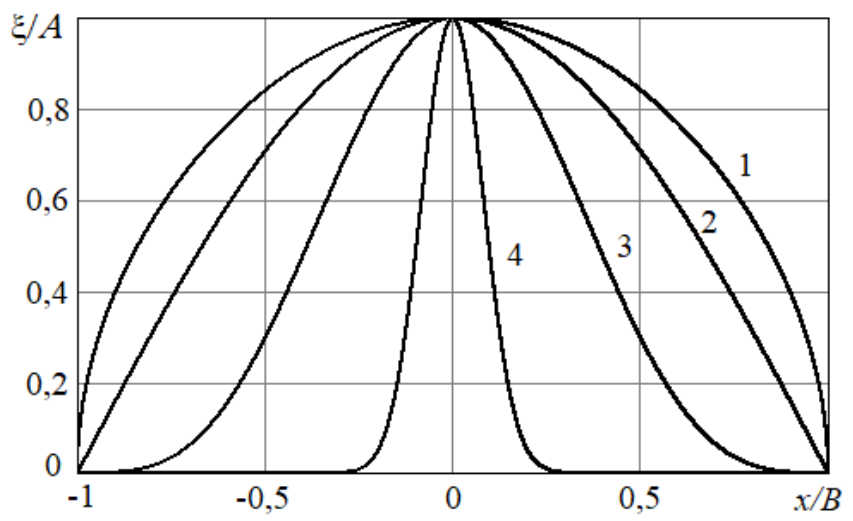

Fig. 7. Profile shape for various parameter values $m: 1-m=0,5 ; 2-m=1 ; 3-m=3,5 ; 4-m=60$.

The analytical expression for the probability density in this case has the form: 


$$
\omega_{\xi}(\xi)=\frac{1}{m A}\left(\frac{\xi}{A}\right)^{\frac{1}{m}-1} \frac{1}{\sqrt{1-\left(\frac{\xi}{A}\right)^{\frac{2}{m}}}} .
$$

The estimation results for losses per unit length in a rectangular waveguide with a cross section of $1.1 \times 0.55 \mathrm{~mm}$ for the profile shape under consideration for various profile heights and parameter $m$ are presented in Fig. 8.

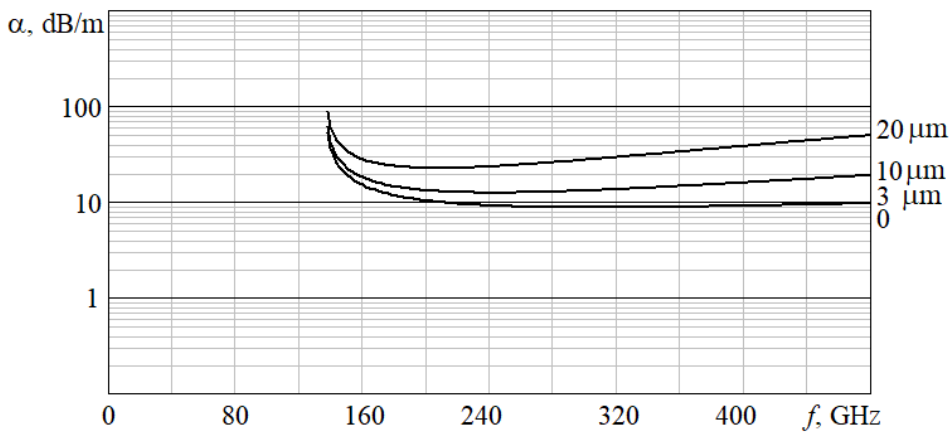

a)

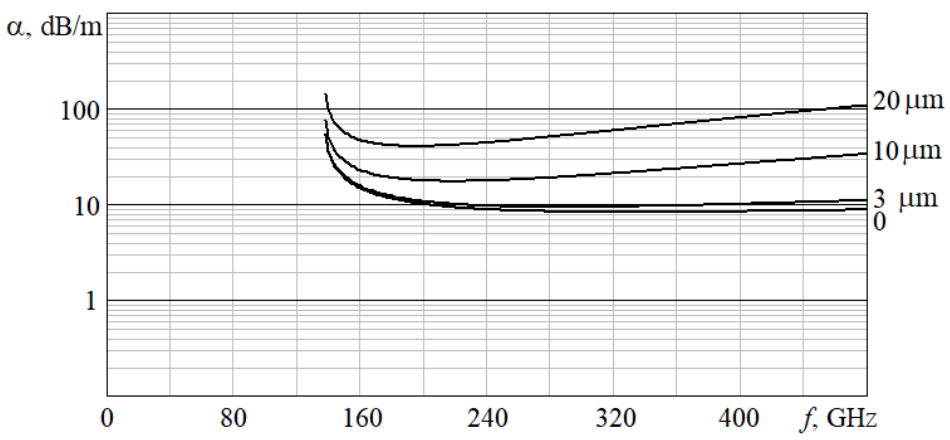

b)

Fig. 8. Dependence on the frequency of losses per unit length in a rectangular waveguide $1,1 \times 0,55$ mm with smooth profile a) $m=0,5$ and $m=60$, b) $m=3,5$.

As follows from these dependences, the attenuation maximum is observed at a parameter $m$ equal to 3.5 .

\section{Conclusion}

An approach to estimating of losses per unit length in a waveguide with rough surfaces is presented; it is based on the concept of partial Brillouin waves and replacing a real rough surface with an equivalent smooth surface.

The estimation of results for the main wave attenuation per unit length in a rectangular waveguide with roughness of various profiles and sizes is presented. The analysis of dependences shows that the height of the screening surface profile roughness has the greatest influence on the natural wave attenuation per unit length. With equal values of the height, the greatest attenuation is observed in the rectangular profile, when the irregularity width is equal to half its period. Application of the proposed method allows setting requirements for the quality of the screening surface processing in the guiding structures, based on the acceptable level of natural wave attenuation. 
The report was prepared on the basis of a study carried out as part of the project of the Russian Science Foundation No. 17-19-01628.

\section{References}

1. R. Ding, L. Tsang, and H. Braunisch, "Random rough surface effects in waveguides using mode matching technique and the method of moments," IEEE Trans. Adv. Packag., accepted, (May 2011)

2. M. Yi et al., "Surface roughness modeling of substrate integrated waveguide in Dband," IEEE Trans. Microw. Theory Techn., vol. 64, no. 4, pp. 1209-1216, Apr. 2016.

3. R. Wang, W. Cui, «A Rapid Estimation of the Conductor Loss in the Rectangular Waveguide With Rough Surface», IEEE (2011)

4. Biryukov, V.V., Estimation of losses in a rectangular waveguide with rough screening surfaces, Antennas, No. 7, P. 53-57 (2016)

5. B. B. Yang, M. P. Kirley, and J. H. Booske, "Theoretical and empirical evaluation of surface roughness effects on conductivity in the terahertz regime," IEEE Trans. THz Sci. Technol., vol. 4, no. 3, pp. 368-375, (May 2014)

6. M. P. Kirley, John H. Booske, «Terahertz Conductivity of Copper Surfaces» IEEE transactions on terahertz science and technology, vol. 5, no. 6, (November 2015) 